\title{
ON ERGODIC MEASURE-PRESERVING TRANSFORMATIONS DEFINED ON AN INFINITE MEASURE SPACE
}

\author{
ARSHAG B. HAJIAN
}

Few, if any, of the properties enjoyed by ergodic measure-preserving transformations defined on a finite measure space generalize in a natural way to those defined on an infinite measure space. Concrete examples of ergodic transformations which preserve a finite measure and ones which preserve an infinite measure exist in the literature, see [1]. It is not difficult to see that ergodic transformations never admit wandering sets of positive measure. In [2] it was shown that a basic difference exists between ergodic transformations which preserve a finite measure and those which preserve an infinite measure; namely, an ergodic measure-preserving transformation defined on an infinite measure space always admits weakly wandering sets of positive measure (Theorem 2 of [2]). Unlike wandering sets, it is not true in general that the union of two weakly wandering sets is again a weakly wandering set even if we require that a class of mutually disjoint images of one weakly wandering set does not intersect a class of mutually disjoint images of the other. One may ask then if there are any ergodic measure-preserving transformations defined on an infinite measure space which admit only weakly wandering sets of finite measure. In this paper we show that this is not the case. We prove that there always exist weakly wandering sets of infinite measure for any ergodic measure-preserving transformation defined on an infinite measure space (Theorem 3). In [2] the existence of a weakly wandering set of positive measure for an ergodic measure-preserving transformation defined on an infinite measure space was discovered while studying the necessary and sufficient conditions for the existence of a finite, invariant, and equivalent measure for a given measurable and nonsingular transformation. In this paper we construct the weakly wandering sets in a different way. Using the pointwise ergodic theorem we prove a simple and yet a useful fact about ergodic measure-preserving transformations defined on an infinite measure space (Theorem 2). It states that given two sets $A$ and $B$ both of finite measure, it is possible to find an image of $A$ under some power of the transformation $T$ which has small intersection with the set $B$. This fact is basic in proving Lemma 1 which shows the existence of a

Received by the editors July 18, 1963. 
special kind of weakly wandering set within any set of finite measure. The main result (Theorem 3 ) then follows from the lemma.

In what follows $(X, B, m)$ will represent a measure space. The whole space $X$ will be measurable, and when we mention an infinite measure space we always assume that it is $\sigma$-finite; that is, $X=\bigcup_{i=1}^{\infty} A_{i}$, where $m\left(A_{i}\right)<\infty$ for $i=1,2, \cdots$. By a measure-preserving transformation $T$ defined on a measure space $(X, B, m)$ we mean a one-toone map of $X$ onto itself such that $m(T B)=m\left(T^{-1} B\right)=m(B)$ for every $B \in B$. $T$ ergodic means that whenever $T A=A$ then either $m(A)=0$ or $m(X-A)=0$. By $L_{1}(X)$ we mean the class of all absolutely integrable, real-valued functions defined on $X$.

The pointwise ergodic theorem states:

TheOREM 1 (G. D. BIRKhofF). Let $T$ be a measure-preserving transformation defined on a finite or an infinite measure space $(X, B, m)$. If $f \in L_{1}(X)$, then the pointwise limit $f^{*}(x)=\lim _{n \rightarrow \infty}(1 / n) \sum_{i=0}^{n-1} f\left(T^{i} x\right)$ exists almost everywhere, $f^{*} \in L_{1}(X), f^{*}(T x)=f^{*}(x)$ almost everywhere, and in case $m(X)<\infty$ we have $\int_{X} f^{*}(x) m(d x)=\int_{X} f(x) m(d x)$.

For a proof we refer the reader to [1].

As a consequence of Theorem 1 we prove

THEOREM 2. Let $T$ be an ergodic measure-preserving transformation defined on an infinite measure space $(X, B, m)$. Let $A$ and $B$ be two sets with $m(A)<\infty$ and $m(B)<\infty$. Then given $\epsilon>0$ there exist arbitrarily large positive integers $n>0$ such that $m\left(T^{n} A \cap B\right)<\epsilon$.

Proof. Given $A$ and $B$ with $m(A)<\infty$ and $m(B)<\infty$, let $f_{A}(x)$ and $g_{B}(x)$ be the characteristic functions of $A$ and $B$, respectively; that is, $f_{A}(x)=1$ if $x \in A$ and equals 0 otherwise, $g_{B}(x)$ is defined similarly. Since $g_{B}(x)$ is in $L_{1}(X)$ it follows from Theorem 1 that $g_{B}^{*}(x)=\lim _{n \rightarrow \infty}(1 / n) \sum_{i=0}^{n-1} g_{B}\left(T^{i} x\right)$ exists almost everywhere; since $g_{B}^{*}(x)$ is an invariant function under the ergodic measure-preserving transformation $T$, we conclude that $g_{B}^{*}(x)=$ constant almost everywhere. From the fact that $g_{B}^{*}(x)$ is an integrable function on $(X, B, m)$ with $m(X)=\infty$ it follows that $g_{B}^{*}(x)=0$ almost everywhere. Now $f_{A}(x)$ is a bounded, non-negative, and integrable function; therefore, applying the bounded convergence theorem and the fact that $(1 / n) \sum_{i=0}^{n-1} f_{A}(x) g_{B}\left(T^{i} x\right)$ converges pointwise to $f_{A}(x) g_{B}^{*}(x)$, we conclude that $\lim _{n \rightarrow \infty}(1 / n) \sum_{i=0}^{n-1} m\left(A \cap T^{-i} B\right)=0$. This implies that $\lim \inf _{n \rightarrow \infty} m\left(T^{n} A \cap B\right)=0$, which proves the theorem.

A measurable set $W$ is said to be weakly wandering (under the transformation $T$ ) if there exists a sequence of integers $\left\{n_{i}: i=0,1,2, \cdots\right\}$ such that $T^{n_{i}} W \cap T^{n_{i}} W=\varnothing$ for $i \neq j$. 
TheOREM 3. Let $T$ be an ergodic measure-preserving transformation defined on an infinite measure space $(X, \mathbb{B}, m)$; then there exists a weakly wandering set of infinite measure.

We first prove a lemma which shows the abundance of a special kind of weakly wandering sets. We note that this lemma is quite similar to Lemma 4 of [2] except that in order to use it in proving the theorem, we state and prove it in the following sharpened version:

LEMMA 1. Let $T$ be an ergodic measure-preserving transformation defined on an infinite measure space $(X, B, m)$. Let $A$ be a measurable set with $0<m(A)<\infty$, and let $\epsilon>0$ be an arbitrary positive number with $\epsilon<m(A)$. Then there exists a subset $A^{\prime}$ of $A$ with $m\left(A^{\prime}\right) \leqq \epsilon$ such that the set $W=A-A^{\prime}$ is weakly wandering under a sequence $\left\{n_{i}: i=0,1,2, \cdots\right\}$. Furthermore, the set $W$ and the sequence $\left\{n_{i}\right\}$ have the following additional property:

(1) $T^{n_{i}+n_{h}} W \cap T^{n_{j+n_{k}}} W=\varnothing$ for $i>h, j>k$, and either $i \neq j$ or $h \neq k$.

Proof. Let $A$ be a measurable set with $0<a=m(A)<\infty$, and $\epsilon>0$ be an arbitrary positive number with $\epsilon<m^{\prime}(A)$. We let $\epsilon_{k}=\epsilon / 2^{k}$ for $k=1,2, \cdots, A_{1}=A$, and $n_{0}=0$. Since $m(A)<\infty$, by Theorem 2 we can choose $n_{1}>0$ such that $m\left(T^{n_{1}} A_{1} \cap A\right)=m\left(T^{n_{1}} A \cap A\right)<\epsilon_{1}$. Next we let $N_{1}=\left\{n_{0}\right\}, N_{2}=\left\{n:-n_{1} \leqq n \leqq n_{1}\right\}$, and $A_{2}=\cup_{i \in N_{2}} T^{i} A$. Since $m\left(A_{2}\right)<\infty$, again by Theorem 2 we can choose $n_{2}>n_{1}$ such that

$$
m\left(T^{n_{2}} A_{2} \cap A\right)=m\left(\bigcup_{i \in N_{2}} T^{n_{2}+i} A \cap A\right)<\epsilon_{2} .
$$

Now suppose that $0<n_{1}<n_{2}<\cdots<n_{k-1}$ have been chosen, we let $N_{k}=\left\{n:-n_{1}-n_{2}-\cdots-n_{k-1} \leqq n \leqq n_{1}+n_{2}+\cdots+n_{k-1}\right\}$, and $A_{k}=\bigcup_{i \in N_{k}} T^{i} A$. Again applying Theorem 2 we can choose $n_{k}>n_{k-1}$ such that

$$
m\left(T^{n_{k}} A_{k} \cap A\right)=m\left(\bigcup_{i \in N_{k}} T^{n_{k}+i} A \cap A\right)<\epsilon_{k} .
$$

Thus we can choose inductively an increasing sequence of positive integers $\left\{n_{k}\right\}$ such that (2) is satisfied for $k=1,2, \cdots$.

Let

Then

$$
A^{\prime}=\bigcup_{k=1}^{\infty} \bigcup_{i \in N_{k}} T^{n_{k}+i} A \cap A
$$

$$
m\left(A^{\prime}\right) \leqq \sum_{k=1}^{\infty} m\left(\bigcup_{i \in N_{k}} T^{n_{k}+i} A \cap A\right) \leqq \sum_{k=1}^{\infty} \epsilon_{k}=\epsilon
$$


Next we show that the set $W=A-A^{\prime}$ has the desired properties. If $i \neq j$, without loss of generality we assume that $i>j$; then since $W=A-A^{\prime}$ and $T^{n_{i}+n_{k}-n_{j}-n_{k}} W \cap A \subset A^{\prime}$, we conclude that

$$
T^{n_{i}+n_{h}-n_{j}-n_{k}} W \cap W=\varnothing .
$$

If $i=j$ and $h \neq k$, again we assume that $h>k$, and since $T^{n_{k}-n_{k}} W \cap A$ $\subset A^{\prime}$, we conclude that

$$
T^{n_{k}-n_{k}} W \cap W=\varnothing .
$$

It is easy to see from the above that

$T^{n_{i+n k}} W \cap T^{n_{j+n_{k}} W}=\varnothing$ for $i>h, j>k$, and either $i \neq j$ or $h \neq k$.

Proof of Theorem 3. By Lemma 1 we construct a set $W$ and a sequence of integers $\left\{n_{i}: i=0,1,2, \cdots\right\}$ which satisfy (2). We let

$$
C=\underset{i \text { even }}{\bigcup} T^{n i} W
$$

Since the sets $T^{n_{i}} W$ for $i$ even are mutually disjoint and $m(W)>0$, we conclude that $m(C)=\infty$. Furthermore, for $h, k$ odd and $h \neq k$ we have

$$
\begin{aligned}
T^{n_{k}} C \cap T^{n_{k}} C & =\bigcup_{i \text { even }} T^{n_{h}+n_{i}} W \cap \underset{j \text { even }}{\bigcup} T^{n_{k}+n_{j}} W \\
& =\bigcup_{i, j \text { even }} T^{n_{i}+n_{k}} W \cap T^{n_{k}+n_{j}} W .
\end{aligned}
$$

The last member of the above equation consists of a countable union of elements of the form $T^{n_{i}+n_{h}} W \cap T^{n_{j}+n_{k}} W$, where $i, j$ are even, $h, k$ are odd, and $h \neq k$.

The following five possible cases are exhaustive:

(i) $i>h, j>k$, and $i \neq j$.

(ii) $i>h, j>k, i=j$, and $h \neq k$.

(iii) $h>i, k>j$, and $h \neq k$.

(iv) $i>h, k>j$, and $i \neq k$.

(v) $h>i, j>k$, and $h \neq j$.

For all the possible cases enumerated above we conclude from (1) that $T^{n_{i}+n_{k}} W \cap T^{n_{j}+n_{k}} W=\varnothing$. This implies that $T^{n_{k}} C \cap T^{n_{k}} C=\varnothing$ fur $h \neq k ; h, k$ odd. This proves that $C$ is a weakly wandering set of infinite measure.

\section{REFERENCES}

1. P. R. Halmos, Lectures on ergodic theory, The Mathematical Society of Japan, 1956.

2. A. B. Hajian and S. Kakutani, Weakly wandering sets and invariant measures, Trans. Amer. Math. Soc. 110 (1964), 136-151.

Cornell University 